\title{
Les lettres de France vues par un Italien du XVI siècle. La bibliothèque française de Lucantonio Ridolfi
}

Le lettere di Francia viste da un italiano del Cinquecento. La biblioteca francese di Lucantonio Ridolfi

French Renaissance Literature Seen through Italian Eyes. The French Books of Lucantonio Ridolfi

\section{Francesco Montorsi}

\section{OpenEdition Journals}

Édition électronique

URL : http://journals.openedition.org/cei/7708

DOI : 10.4000/cei. 7708

ISSN : 2260-779X

Éditeur

UGA Éditions/Université Grenoble Alpes

Édition imprimée

ISBN : 978-2-37747-225-3

ISSN : 1770-9571

\section{Référence électronique}

Francesco Montorsi, « Les lettres de France vues par un Italien du xvi e siècle. La bibliothèque française de Lucantonio Ridolfi », Cahiers d'études italiennes [En ligne], 31 | 2020, mis en ligne le 06 octobre 2020, consulté le 27 mars 2021. URL : http://journals.openedition.org/cei/7708 ; DOI : https://doi.org/ $10.4000 /$ cei.7708

Ce document a été généré automatiquement le 27 mars 2021

(c) ELLUG 


\title{
Les lettres de France vues par un Italien du XVI ${ }^{\mathrm{e}}$ siècle. La bibliothèque française de Lucantonio Ridolfi
}

\author{
Le lettere di Francia viste da un italiano del Cinquecento. La biblioteca francese \\ di Lucantonio Ridolfi
}

French Renaissance Literature Seen through Italian Eyes. The French Books of Lucantonio Ridolfi

Francesco Montorsi

$1 \quad$ Les lettres italiennes du XVI italien se caractérisent par un certain désintérêt envers les élaborations littéraires provenant d'au-delà des Alpes. À une époque où les modèles de la Péninsule pénètrent toute la culture européenne et jouissent d'un prestige absolu, le discours italien fait une place toute relative aux modèles culturels venus de l'étranger. Un certain mépris, particulièrement aigu au début et au milieu du siècle, accompagne le traitement de la situation littéraire française. Dans L'olive, Joachim Du Bellay évoque ainsi «le peu d'estime que font les Italiens de nostre poesie en comparaison de la leur ${ }^{1} »$.

2 Malgré le désintérêt italien d'ensemble pour les productions transalpines, des exceptions, parfois significatives, se signalent néanmoins. Dans bien des cas, elles concernent des hommes qui, grâce à leur parcours biographique - notamment des séjours, voire une résidence durable au-delà des Alpes - ont acquis une connaissance approfondie des lettres de France. L'un de ceux-ci est Lucantonio Ridolfi, de noble famille florentine, dont la résidence à Lyon est attestée à partir de la deuxième moitié des années $1530^{2}$.

3 En ce début du $\mathrm{XVI}^{\mathrm{e}}$ siècle, ce Lyon où habite Ridolfi est une ville "italienne » ou «italianisée ». Un vaste contingent de transalpins fortunés, marchands et banquiers, y ont pris résidence et profitent des riches foires qui ont lieu quatre fois par an. En raison 
de sa colonie étrangère mais également de son emplacement géographique, Lyon subit l'influence des modèles italiens ${ }^{3}$.

4 Dans cette ville, Lucantonio Ridolfi s'adonne aux activités du négoce et de la culture. Il fait partie d'un cercle littéraire où se trouvent Italiens et Français sensibles aux nouveautés venues d'au-delà des Alpes. Il côtoie Maurice Scève et Pontus de Tyard, et collabore avec Guillaume Rouillé. Ce dernier est un libraire italianisant, à une époque où la ville du Rhône est un important centre de production du livre italien ${ }^{4}$.

5 L'activité littéraire de Ridolfi s'exerce dans plusieurs traductions du latin en langue italienne demeurées manuscrites. L'édition attire son intérêt aussi. Entre 1550 et 1555, il participe, suivant des modalités différentes et parfois peu claires, à la publication d'éditions des trois couronnes chez Rouillé. Il publie aussi deux œuvres originales. Ce sont deux traités en forme de dialogue parus encore une fois chez Rouillé, en 1557 et en 1560 .

6 La présente contribution vise à retrouver les lectures françaises de cet homme de plume à cheval entre deux pays et deux cultures. En l'absence de l'inventaire de l'ancienne bibliothèque de Ridolfi, nous choisissons de reconstruire celle-ci en ayant recours à des indices, c'est-à-dire les renvois aux textes français glissés par Ridolfi luimême dans son œuvre. Ce n'est donc pas une bibliothèque réelle qui nous occupera ici, mais son image, certes fragmentaire et sélective, telle qu'elle est dessinée par la plume d'un lecteur qui est aussi écrivain.

7 L'analyse va porter en particulier sur les œuvres originales de Ridolfi, deux traités en forme de dialogue. Le premier s'intitule, si l'on reprend le titre dans son intégralité :

Ragionamento havuto in Lione, da Claudio de Herberè gentil'huomo franzese, et da Alessandro degli Uberti gentil'huomo fiorentino, sopra alcuni luoghi del cento novelle di Boccaccio. I quali si ritroveranno secondo i numeri delle Carte del Decamerone stampato in Lione in piccola forma da G. Rovillio, l'Anno M.D.LV

Il s'agit d'un dialogue fictif entre un Florentin, Alessandro degli Uberti, et un Français expert de littérature italienne, Claude de Herberay (le nom va revenir dans l'analyse et doit être retenu). Le débat touche de nombreuses questions philologiques et linguistiques soulevées par le chef-d'œuvre de Boccace. L'édition de 1555 évoquée dans le titre a été préparée par Rouillé en collaboration avec Ridolfi lui-même.

Le deuxième traité aurait été composé également en 1556-1557, ainsi que le montrent la date de la lettre de dédicace et d'autres indices ${ }^{7}$. Il a été publié en 1560. Le dialogue a pour titre :

Aretefila. Dialogo nel quale da una parte sono quelle ragioni allegate, le quali affermano, lo amore di corporal bellezza potere ancora per la via dell'udire pervenire al quore: et dall'altra, quelle che vogliono lui havere solamente per gl'occhij l'entrata sua: colla sentenza sopra cotal quistione.

10 Ce dernier ouvrage fournit le plus grand contingent d'exemples pour notre travail, ce pourquoi il doit être décrit plus en détail. Le texte met en scène un débat ayant lieu dans le salon lyonnais d'une dame qui se cache derrière le pseudonyme d'Aretefila, d'après une étymologie grecque qui veut dire "Celle qui aime la vertu». Outre cette dame, prennent part à la discussion deux gentilshommes toscans, Federico qui vient d'arriver d'Italie, et Lucio, proche de la maitresse des lieux et résident à Lyon. Ridolfi présente le texte comme la transcription d'un dialogue réel qui lui a été rapporté par l'un des participants. Il précise toutefois qu'il a pris soin de cacher l'identité des personnages. 
11 Malgré ces précautions, l'identité de deux protagonistes sur trois se laisse percer: Aretefila est Marguerite de Bourg, figure de proue de l'italianisme lyonnais ; Lucio est l'alter ego de l'auteur lui-même Lucantonio Ridolfi ${ }^{9}$. Federico, dont le contexte ne fournit aucune identification, pourrait relever d'une persona fictive, dont le but narratif est de s'opposer aux raisons de Lucio. Le dialogue, si tant est que le récit de Ridolfi reflète une situation réelle, doit s'être déroulé à l'hiver 1556-1557, peu après le 14 novembre $1556^{10}$.

12 Le dialogue affronte une questione d'amore, à savoir si cet amour qu'en italien on appelle innamoramento per fama existe bien ${ }^{11}$. Federico essaie d'en démontrer l'existence, alors que Lucio - que l'on doit identifier avec l'auteur du dialogue lui-même - s'oppose à cette thèse. L'ouvrage s'inscrit donc dans la tradition des débats d'amour, qui bénéficie en Italie d'un ample succès depuis le commentaire de Ficin du Banquet en 1468 et qui voit au $\mathrm{XVI}^{\mathrm{e}}$ siècle d'importantes contributions, comme celle des Dialoghi d'amore de Léon l'Hébreu ou encore des traités de Mario Equicola et Bembo.

13 L'Aretefila a attiré l'attention des chercheurs en raison du témoignage que le dialogue porte sur le milieu italianisant au $\mathrm{XvI}^{\mathrm{e}}$ siècle. En effet, le texte illustre les relations étroites que Marguerite de Bourg, alias Aretefila, entretient avec des hommes de lettres tels que Benedetto Varchi et Luigi Alamanni. Celle-ci déploie une connaissance approfondie de la littérature italienne ancienne et contemporaine. Aretefila cite, souvent avec force éloges, de nombreux contemporains - tels que Giovanni Della Casa, Pietro Bembo, Annibal Caro, Luigi Alamanni, Giovanni Andrea Dell'Anguillara, Benedetto Varchi - sans compter les trois couronnes Dante, Pétrarque et Boccace, ou encore Léon l'Hébreu ${ }^{12}$.

Or le texte contient également d'autres références, des allusions à des auteurs classiques $^{13}$, et aussi à des textes français. Ce sont ces derniers qui vont être au centre de l'analyse. Grâce au dépouillement des dialogues de Ridolfi, il a été possible d'établir un relevé de récits, traités et recueils poétiques cités par les devisants. Le tableau suivant énumère les ouvrages et les auteurs dans l'ordre de parution dans le texte, chaque entrée étant accompagnée du nom du personnage qui en fait mention. L'Aretefila fournit toutes les occurrences avec une seule exception, tirée du Ragionamento.

Textes français cités par Lucantonio Ridolfi :

\begin{tabular}{|l|}
\hline Pontus de Tyard, Discours du temps, de l'an et de ses parties [p. 13-14 Lucio et Aretefila]. \\
\hline François de Billon, Le fort inexpugnable de l'honneur du sexe féminin [p. 14 Lucio]. \\
\hline $\begin{array}{l}\text { Pontus de Tyard (tr.), Léon L'Hébreu, De l'Amour [p. } 15 \quad \text { Aretefila ; p. 56-58 Federico; } \\
\text { p. } 117 \text { Aretefila]. }\end{array}$ \\
\hline Pontus de Tyard, Premier Solitaire et Second Solitaire [p. 15 Lucio]. \\
\hline Tristan [p. 38 Federico]. \\
\hline Primaléon de Grèce [p. 38-39 Federico]. \\
\hline Pierre de Provence et la Belle Maguelonne [p. 39 Federico; p. 40-43 Aretefila]. \\
\hline
\end{tabular}




\begin{tabular}{l} 
Raimbaut d'Orange et Jaufré Rudel [p. 45-47 Federico]. \\
\hline Amadis de Grèce [p. 44 Federico ; p. 52 Federico ; p. 61-62 Federico ; p. 73 Federico]. \\
\hline Héliodore, L'Histoire aethiopique [p. 52-53 Federico; p. 70-71 Federico]. \\
\hline Maurice Scève, ses traductions et imitations de Pétrarque [p. 135-136 Aretefila et Lucio]. \\
\hline Mellin de Saint-Gelais, premier inventeur du sonnet en France [p. 137 Aretefila]. \\
\hline Clément Marot, premier traducteur de « cose latine et toscane » en français [p. 137 Aretefila]. \\
\hline $\begin{array}{l}\text { Pontus de Tyard, Ronsard et Du Bellay, premiers inventeurs de sonnets en France et auteurs de } \\
\text { recueils poétiques excellents [p. 137 Aretefila]. }\end{array}$ \\
\hline Maurice Scève, Délie et Blason du Cil [p. 138-143 Aretefila, Lucio et Federico]. \\
\hline La Mort le roi Arthur [Claudio Herberay dans Ragionamento, p. 43-45].
\end{tabular}

La bibliothèque ainsi reconstituée est composée de 16 items, qui correspondent à neuf auteurs français, trois récits anonymes et quatre textes étrangers (un italien, deux castillans, un grec, mais sont aussi mentionnées des traductions et imitations de l'italien non précisées). Du point de vue du genre, nous avons une certaine variété : des recueils poétiques, des traités, des fictions en prose. La présence significative de romans ne sera pas sans surprendre certains, d'autant plus que cinq sur six sont des récits de chevalerie, d'origine à la fois médiévale et moderne. Certains auteurs et œuvres sont cités une seule fois, d'autres plusieurs. Le poète Pontus de Tyard et l'Amadis de Grèce sont évoqués à quatre reprises.

Le Florentin Ridolfi présente un tableau informé sur la production poétique de son époque. Il évoque la génération de Clément Marot et Mellin de Saint-Gelais, la Pléiade de Ronsard et Du Bellay ainsi que l'école lyonnaise de Maurice Scève et Pontus de Tyard. Plusieurs œuvres sont très récentes - Pontus de Tyard, Du Bellay et Ronsard publient leurs chansonniers en 1549 et 1552 - et nous avons là l'une de leurs toutes premières mentions italiennes. La plupart de ces auteurs sont cités dans une discussion sur l'introduction du sonnet en France, un passage qui constitue l'un des plus importants témoignages contemporains sur la question et qui mérite à ce titre d'être ici reporté intégralement.

Aretefila exprime son regret que Maurice Scève ne soit pas présent au débat. Il l'aurait illustré, dit-elle, avec son savoir et aurait soulagé la tâche des devisants. Scève aurait d'ailleurs accepté l'invitation, nous dit-on, car il est autant courtois que savant et il aime grandement la nation italienne et sa littérature, ce pourquoi il a traduit et imité nombre de sonnets de Pétrarque. Cette observation est l'occasion d'introduire une question de Lucio, qui donne le jour à un bref historique sur l'introduction du sonnet en France :

[Lucio] «Mais répondez-moi : est-ce que Mons. Scève fut le premier qui introduisit dans votre langue cette manière de versification qui en notre langue s'appelle sonnet?»

[Aretefila] « Non, - répondit-elle - bien qu'il ait traduit d'innombrables sonnets de votre langue, ainsi que je l'ai dit, et qu'il ait composé dans notre langue de très 
nombreux sonnets de son invention avec savoir et grâce inégalable. Mais je crois que le premier fut quelqu'un qui s'appelle Monseigneur de Saint Gelais [i.e. Mellin de Saint-Gelais], faiseur de rimes très remarquable, et dont on a vu de très nombreuses et excellentes compositions, bien qu'il en ait fait imprimer très peu. Il est vrai que le premier qui traduisit des choses latines et toscanes en notre langue française fut, je crois, Clément Marot. Mais en ce qui concerne le sonnet, non seulement ces deux que je vous ai nommés l'ont transporté en notre langue et appelé avec le même nom que vous, mais de nombreux autres l'ont fait. Parmi ceuxci, pour l'instant je ne veux que vous en citer trois excellents, bien que les autres soient aussi dignes de très grande louange. Ce sont Tyard, Ronsard et du Bellay, dont on lit, imprimées en notre langue, trois œuvres poétiques qui font resplendir les noms de leurs auteurs - ce pourquoi il n'est pas besoin de multiplier leurs louanges, bien que Mons. de Tyard ait imité davantage Pétrarque que les autres, puisqu'il a composé avec grand savoir, outre les sonnets, de nombreuses chansons et sextines. Or, je ne voudrais pas, ajouta Aretefila, que le récit de choses aussi élevées me fasse sortir de mon propos, qui est d'exprimer la gloire que Mons. Scève s'est légitimement acquise au moyen de ses compositions. Ce qui mérite bien, Frédéric, que vous ayez la patience d'écouter ${ }^{14}$. »

Ridolfi ne cite pas les œuvres elles-mêmes, mais se réfère à des auteurs, ce pourquoi nous ne trouvons pas les titres L'Olive, Livre des amours, Erreurs amoureuses, mais seulement les noms des auteurs Du Bellay, Ronsard, de Tyard. En revanche, peu après les devisants renouvellent les éloges à Maurice Scève - déjà encensé par Aretefila - en vantant la beauté de poèmes et d'œuvres précis, tels Délie et le Blason du Cil. L'autre poète lyonnais reçoit, tout comme Scève, une mention d'honneur - modestement moulée dans une phrase à tournure concessive. En effet, quant à la composition du sonnet, Pontus de Tyard mérite davantage de considération que Du Bellay et Ronsard, nous dit-on, puisqu'il a mieux suivi le modèle de Pétrarque. Au début du dialogue, Ridolfi, sous le masque de Lucio, avait déjà eu des mots de louange pour Pontus de Tyard à propos de ses traités (Discours du temps, de l'an et de ses parties, Premier Solitaire, Second Solitaire) et de sa traduction de Léon l'Hébreu (Dialogues d'amour). L'un de ces textes de Pontus de Tyard avait été évoqué non pas pour lui-même mais dans le cadre d'un hommage à Aretefila : Lucio avait alors rappelé que le Discours du temps était dédié à Aretefila, en dévoilant ainsi la coïncidence entre celle-ci et Marguerite de Bourg. La citation du Fort inexpugnable, traité féministe de François de Billon, s'explique également par l'éloge de la puissante dame. Tous ces récits font parties des nouveautés littéraires, ayant paru en 1551 et 1552 (traduction d'Hébreu et Premier solitaire; Second Solitaire), en 1555 (Fort inexpugnable) et même en 1556 (Discours du temps), c'est-à-dire peu de mois avant le débat. Mellin de Saint-Gelais est connu à la fois dans sa tradition imprimée, peu vaste à cette date, mais surtout dans ses témoins manuscrits, ce qui révèle l'accès de Ridolfi aux milieux culturels français où ces œuvres circulaient.

On remarquera que la tradition littéraire française est citée essentiellement par le biais d'un filtre particulier, celui de l'italianisme. Mellin de Saint-Gelais et Clément Marot sont évoqués non pas pour leur œuvre, mais pour leur rôle pionnier dans l'innutrition de modèles transalpins, acculturation qui semble s'achever, selon Ridolfi, avec la génération de Pontus de Tyard, Ronsard, Du Bellay et Scève. Soulignons enfin que les devisants ne parlent pas des poètes des anciennes générations, à une exception près - toute relative - celle de deux troubadours, évoqués par le biais de Pétrarque ${ }^{15}$.

La «bibliothèque » française de Ridolfi n'est pas composée seulement de poètes, mais aussi de fictions en prose. Dans ce domaine, on trouve le roman grec d'Héliodore 
(L'Histoire aethiopique, appelé aussi Les Amours de Théagène et Chariclée) et cinq récits de chevalerie. Parmi ces derniers, les quatre abordés dans l'Aretefila - Amadis, Primaléon de Grèce, Tristan, Pierre de Provence - sont évoqués dans un espace très bref par Federico qui les introduit en disant :

Je vais continuer mon raisonnement en vous racontant certains exemples qui se lisent dans vos romans français [ne' vostri romanzi francesi], tels que je les ai entendus raconter par une personne digne de confiance ${ }^{16}$.

21 L'Histoire aethiopique est citée, quant à elle, deux fois dans l'Aretefila, les deux fois pour des questions « annexes", non par pour en tirer des exemples ou pour vanter la beauté de l'œuvre.

À l'instar de ce que l'on observait dans le domaine lyrique, nous avons affaire là à des œuvres originales et à des traductions. D'un côté, des textes français médiévaux (Tristan, La Mort le roi Arthur, Pierre de Provence). De l'autre des libros de caballerías (Amadis, Primaléon de Grèce) et une traduction du grec (L'Histoire aethiopique). Les traductions françaises d'Amadis et de l'Histoire aethiopique, dues à Nicolas Herberay des Essarts et Jacques Amyot, sont célèbres à l'époque. De 1540 à 1548, Herberay des Essarts acquiert une vaste renommée en traduisant la saga d'Amadis, du livre I jusqu'aux livres VII et VIII (l'Amadis de Grèce). L'Histoire aethiopique traduite par l'humaniste Jacques Amyot devient un classique dès l'instant de sa publication en 1548. Par ailleurs, dans le lot des titres en prose, nous avons affaire à la fois à des ouvrages antiques, modernes et médiévaux. L'intérêt manifesté par Ridolfi envers les récits chevaleresques - cinq des six titres - est remarquable. Bien représentée dans l'Aretefila, cette curiosité est confirmée dans le Ragionamento par la référence à la bataille mortelle entre Arthur et Mordred, épisode qui se trouve originellement dans La Mort le roi Arthur et ensuite dans le Lancelot imprimé à partir de 1488. On aura également constaté que le nom de l'un des deux devisants du Ragionamento, Claudio de Herberé, semble renvoyer au célèbre traducteur des Amadis, à moins qu'il ne s'agisse d'un personnage historique inconnu par ailleurs de la critique.

Alors que les poètes français - de Pontus à Scève, de Marot à Ronsard - sont évoqués pour souligner leur talent artistique ou leur parfaite imitation de modèles italiens, les références aux fictions en prose ont un autre but. Elles servent de réservoir d'exemples, en appui à la thèse de l'amour de loin. C'est le tenant de celle-ci, Federico, qui mobilise le domaine, à deux exceptions près, dont celle déjà citée de La Mort le roi Arthur. Pierre de Provence est évoqué, quant à lui, par Lucio mais ensuite Aretefila prend soin de le résumer longuement. Ému par le récit de la dame, Lucio finit par comparer le texte aux nouvelles de la cinquième journée du Décaméron.

On pourrait trouver surprenant de voir dans le même traité le nom de Ronsard et des romans tels que Tristan ou l'Amadis. C'est que des modèles historiographiques anciens, souvent critiqués mais jamais dépassés, négligent le rôle joué par la fiction chevaleresque dans la culture renaissante. Les récits médiévaux d'abord et les romans castillans ensuite sont à cette époque une lecture typique des classes aisées, entre autres nobiliaires. Le roman de chevalerie sert comme moyen traditionnel de formation aristocratique. Par ailleurs, le genre chevaleresque renaissant participe au projet d'illustration de la langue et la culture françaises, qui sera plus tard associé à la Pléiade. Avant la condamnation par les doctes, l'entreprise de Nicolas Herberay des Essarts est considérée comme le sommet de la prose française. Il en va ainsi dans les années 1540, alors que dans les années 1550 les critiques se font plus fréquentes et le 
roman, malgré l'énorme fortune de l'Amadis entre autres, devient un genre qui doit être défendu ${ }^{17}$.

Ridolfi ne cite pas seulement ce grand succès qu'est l'Amadis, mais évoque aussi l'autre livre de fiction qui suscite l'enthousiasme dans les années 1540. L'Histoire aethiopique du grec Héliodore est traduite par l'humaniste et futur évêque Jacques Amyot en 1548. Ce texte, présenté comme un antiroman de chevalerie en raison de sa forme classique et de sa haute moralité, est vu comme un chef-d'œuvre de la prose par les lecteurs français ${ }^{18}$. Lucantonio Ridolfi semble donc citer les plus importantes nouveautés de la fiction en prose de l'époque - des ouvrages comme les Amadis et l'Histoire aethiopique à côté de textes plus vieux, mais toujours courants, comme les récits chevaleresques médiévaux.

À la différence des recueils lyriques, les romans - qui servent à fournir des exemples pour le débat - sont évoqués de manière précise par les devisants (en particulier Federico). Le lecteur trouve ainsi des renvois à des épisodes particuliers. Nous avons cru utile de comparer ces transpositions du dialogue italien avec les morceaux originels qui se lisent dans les fictions en prose elles-mêmes. Ce travail nous a amené à mieux comprendre les parcours de lecture propres à notre auteur. Contrairement à ce que semble indiquer Federico, Ridolfi connaît et cite les récits de chevalerie castillans et le roman grec d'Héliodore d'après leurs traductions italiennes, et non pas dans leurs versions françaises.

La démonstration fonctionne comme un syllogisme. L'Amadis de Grecia de Feliciano de Silva a paru en un seul volume à Cuenca en $1530^{19}$. En France, il a été traduit par Nicolas Herberay des Essarts en deux volumes, en 1546 et en 1548. Il s'agit des Amadis VII et $V I I I^{20}$. Il est essentiel de noter que les toutes dernières pages de l'Amadis de Grecia espagnol ont été exclues de l'Amadis VIII. Par contre, elles ont été reprises par le volume suivant, paru en 1551, un ouvrage qui ne s'appelle plus Amadis de Grèce, mais Florisée de Niquée $^{21}$. Le choix d'intégrer les derniers chapitres dans un livre ultérieur s'explique par le fait que ces épisodes finaux constituent un hors d'œuvre par rapport à la trame de l'Amadis de Grecia et préparent précisément la suite de la saga.

Dans Aretefila, l'Amadis de Grecia est l'un des textes les plus mis à contribution, avec l'évocation de quatre morceaux distincts. Si l'on regarde maintenant dans quels livres français se trouvent les quatre passages cités par Federico, on constate qu'ils se lisent dans les livres VII et VIII, mais aussi au début du livre IX. Donc dans les deux volumes qui s'appellent Amadis de Grèce mais aussi dans le livre qui en français prend le nom de Florisée $e^{22}$.

Or, dans l'Aretefila, Federico cite les quatre épisodes sous un seul titre, celui déjà originel d'Amadis de Grèce. Cela veut dire qu'il n'a pas lu le roman dans sa version française. L'aurait-il lu dans le texte castillan ? Cela paraît peu vraisemblable, d'autant que Ridolfi ne cite pas d'autres auteurs espagnols. Il l'aura par conséquent connu dans sa traduction italienne, qui avait, elle, fidèlement rendu le volume de Feliciano de Silva, et qui était disponible depuis $1550^{23}$.

Les conséquences de ces déductions peuvent s'étendre selon nous au cas représenté par l'autre libro de caballerías cité par Federico, le Primaléon. Ce récit aura aussi été lu dans sa version italienne. Remarquons en passant que, bien qu'ils n'aient pas été traduits par un lettré de l'envergure d'un Herberay des Essarts et qu'ils n'aient pas joui d'un engouement critique, les récits de chevalerie castillans rencontraient au milieu du 
siècle un vaste succès auprès du public en Italie, où ils sont réimprimés à de nombreuses reprises ${ }^{24}$.

31 La démonstration d'une filiation italienne est plus facile à faire pour la traduction d'Héliodore. Le roman grec est évoqué deux fois dans Aretefila, une fois à propos de certaines coutumes des Grecs (p. 70-71), une autre fois pour en extraire une citation, en italien, qui sert d'appui à un exemple (p. 52-53). Celle-ci ne correspond pas à la version française d'Amyot qui aurait été traduite pour l'occasion du français à l'italien et, se trouve, en revanche, de manière presque identique, dans la version italienne de Leonardo Ghini publiée en 1556 :

\begin{tabular}{|c|c|c|}
\hline Aretefila & Traduction de Ghini & Traduction d'Amyot \\
\hline $\begin{array}{l}\text { Et appresso Heliodoro delle cose } \\
\text { Etiopiche si leggono queste parole: o } \\
\text { padre, questo suo ragionamento gli } \\
\text { ha così efficacemente espressi che io } \\
\text { gli contemplava, ancora che assenti } \\
\text { et non vedendogli mi pareva vedergli } \\
\text { (p. 52-53). }\end{array}$ & $\begin{array}{l}\text { E Cnemone; o padre questo } \\
\text { tuo ragionare gli ha così } \\
\text { efficacemente espressi, che } \\
\text { io gli contemplava anchor } \\
\text { che assenti; e non } \\
\text { vedendogli mi pareva } \\
\text { vedergli }{ }^{25} \text {. }\end{array}$ & $\begin{array}{l}\text { Il m'estoit encore avis (mon } \\
\text { pere) respondit Gnemon, que } \\
\text { je les voyois, encore qu'ilz } \\
\text { fussent absents, tant vostre } \\
\text { dire me les a clairement } \\
\text { representez tous telz que je } \\
\text { les ay veuz }{ }^{26} \text {. }\end{array}$ \\
\hline
\end{tabular}

Notre relevé se conclut sur les récits, à la fois médiévaux et français, de Tristan et Pierre de Provence. Le premier est cité par Ridolfi de manière énigmatique pour un épisode qui ne s'y trouve pas, au moins dans les versions françaises ${ }^{27}$. Le deuxième fait l'objet d'un long et élogieux résumé de la part du personnage Aretefila. Imprimé à d'innombrables reprises en France et cité par des poètes tel que Marot (auteur de "L'Épître de Maguelonne»), Pierre de Provence ne semble pas être passé à l'imprimé en langue italienne aux $\mathrm{XV}^{\mathrm{e}}$ et $\mathrm{XVI}^{\mathrm{e}}$ siècles et il est donc presque sûr que Ridolfi l'a lu dans les éditions publiées en ces années à Lyon et à Paris. En revanche, cela ne peut pas être avancé pour le Tristan, qui a été publié sept fois à Paris entre 1489 et 1533, mais que Ridolfi pourrait avoir connu dans l'une de ses déclinaisons italiennes.

En conclusion, le cas de Lucantonio Ridolfi constitue un témoignage extraordinaire de la réception italienne de la littérature française pendant la Renaissance. À une époque où les belles-lettres de France occupent peu les esprits de la Péninsule, le marchand toscan déploie une solide connaissance de textes transalpins, lyriques et narratifs, connus à la fois par les imprimés et, pour le cas de Mellin Saint-Gelais, par la tradition manuscrite. La photographie de la littérature élaborée par Ridolfi frappe par son caractère d'actualité. Le dialogue qui a donné le jour à l'Aretefila aurait eu lieu pendant l'hiver 1556-1557 alors que les devisants évoquent des œuvres très récentes, publiées pour certaines en 1555 et 1556.

Ridolfi nomme les poètes plus importants de ces années centrales du siècle. Ces hommes de lettres proviennent de différents horizons culturels, et on trouve citée l'école de Clément Marot et Mellin de Saint-Gelais à côté de la Pléiade de Ronsard et $\mathrm{Du}$ Bellay. Les choix littéraires du marchand reflètent aussi ses allégeances. Les renvois à Maurice Scève et à Pontus de Tyard sont un hommage affectueux au milieu lyonnais que Ridolfi fréquente avec Marguerite de Bourg. Le filtre national joue un rôle dans la 
sélection proposée : c'est la question de l'imitation des modèles italiens qui ouvre la voie à un long passage de l'Aretefila où sont passés en revue les principaux poètes français de l'époque.

Des textes en prose, dont des récits de chevalerie, sont l'objet de citation. C'est d'autant plus remarquable que nous avons affaire là à un genre qui malgré son ancien prestige est, en ces années, de plus en plus critiqué. À cet égard, on pourra remarquer que les citations proviennent, dans leur grande partie, de Federico - celui même à qui s'oppose l'alter ego de l'auteur, Lucio, vainqueur du débat. Par ce biais, Ridolfi affiche son désintérêt, qu'il soit sincère ou pas, à l'égard d'un genre frappé par les accusations des doctes.

Les citations des récits en prose posent par ailleurs un problème textuel. Dans l'Aretefila sont évoqués entre autres l'Amadis de Grecia et l'Histoire aethiopique d'Héliodore. L'analyse a montré que l'auteur puise ses matériaux à partir des transpositions italiennes de ces textes, alors que son dialogue semble faire allusion aux versions françaises. Ce sont donc des citations en trompe-l'œil. Ce geste s'explique par l'une des finalités narratives propres à l'entreprise de Ridolfi, celle d'illustrer l'actualité du monde éditorial de son époque.

Il revient ainsi à Lucantonio Ridolfi - Italien de nation, Lyonnais d'adoption l'honneur d'avoir brossé, dans son Aretefila, l'un des panoramas les plus lucides des lettres françaises des années 1540 et 1550. C'est-à-dire un tableau où se côtoient les plus importants poètes de la génération du milieu du siècle - Scève, Ronsard, de Tyard, Du Bellay, Marot, Saint-Gelais -, ainsi que des textes tels que l'Amadis et l'Histoire aethiopique, c'est-à-dire à la fois les principaux modèles de la prose narrative comme ceux de la poésie lyrique.

\section{BIBLIOGRAPHIE}

BALMAS Enea, Ronsard et l'Italia: una dialettica antica, dans Ronsard e l'Italia. Ronsard in Italia, Atti del 1 Convegno del Gruppo di Studio sul Cinquecento francese, Fasano, Schena, 1988.

BiANCHI Natascia, « Un altro lettore di Dante nel Cinquecento », Studi Danteschi, n 66, 2001, p. 205-223

COOPER Richard, « Le Cercle de Lucantonio Ridolfi », dans M. Clément et J. Incardona (éds), L'Émergence littéraire des femmes à Lyon à la Renaissance, Saint-Étienne, Publications de l'Université de Saint-Étienne, 2008, p. 29-50.

DionisotTi Carlo, Geografia e storia della letteratura italiana, Turin, Einaudi, 1967.

Dusi Nicola, «Lucantonio Ridolfi e Francesco Petrarca: un esegeta fiorentino a Lione ", Studi Petrarcheschi, $\mathrm{n}^{\circ}$ 20, 2007, p. 125-150.

FAVARo Maiko, « Boccaccio nella trattatistica amorosa del Cinquecento e di primo Seicento ", Nuova Rivista di Letteratura Italiana, vol. XII, n 1-2, 2009, p. 9-29. 
FonTAINE Marie Madeleine, « “Un cœur mis en gage”. Pontus de Tyard, Marguerite de Bourg et le milieu lyonnais des années 1550 », Nouvelle Revue du XvI siècle, vol. II, 1984, p. 69-89.

GiUdicI Enzo, « Luc'Antonio Ridolfi et la Renaissance franco-italienne », Quaderni di filologia e lingue romanze, n. s., vol. I, 1985, p. 111-150.

LESAGE Claire, «"La forma del ben dire" : les lecteurs français du Ragionamento de Lucantonio Ridolfi (1557) », dans P. Guérin et A. Robin (éds), Boccaccio e la Francia, Florence, Franco Cesati, 2017, p. 259-270.

PICоT Émile, Les français italianisants au XVI siècle, Paris, Champion, 1906-1907, 2 vol.

Procaccioli Paolo, « Le "tre corone" a Lione. Guillaume Rouville e Lucantonio Ridolfi », dans S. D'Amico et S. Gambino Longo (éds), Le savoir italien sous les presses lyonnaises à la Renaissance, Genève, Droz, 2017, p. 223-244.

[RIDOLFI Lucantonio], Ragionamento havuto in Lione, da Claudio de Herberè gentil'huomo franzese, et da Alessandro degli Uberti gentil'huomo fiorentino, sopra alcuni luoghi del cento novelle di Boccaccio. I quali si ritroveranno secondo i numeri delle Carte del Decamerone stampato in Lione in piccola forma da G. Rovillio, l'Anno M.D.LV, in Lione, appresso Guillaume Rouillé, 1557.

RiDOLFI Lucantonio, Aretefila. Dialogo nel quale da una parte sono quelle ragioni allegate, le quali affermano, lo amore di corporal bellezza potere ancora per la via dell'udire pervenire al quore: et dall'altra, quelle che vogliono lui havere solamente per gl'occhij l'entrata sua: colla sentenza sopra cotal quistione, in Lione, appresso Guliel. Rovillio, M.D.LX.

TRAVERSA Rosanna, L'attività lionese di Luc'Antonio Ridolfi, Tesi di laurea, Università di Torino, 1964-1965.

\section{NOTES}

1. Joachim Du Bellay, L’Olive, «Au lecteur », voir CEuvres poétiques, t. I, D. Aris et F. Joukovsky (éds), Paris, Garnier, 2009, p. 10. Pour l'indifférence du $\mathrm{XVI}^{\mathrm{e}}$ siècle italien envers les belles-lettres françaises, voir Carlo Dionisotti (Geografia e storia della letteratura italiana, Turin, Einaudi, 1967, p. 172-173, où le critique parle d'« un disinteresse in Italia per quel che si scrive[va] o pubblica[va] in Francia tanto maggiore quanto più forte era l'influsso italiano sulla cultura francese ») et aussi E. Balmas, "Ronsard et l'Italia: una dialettica antica», dans Ronsard e l'Italia. Ronsard in Italia, Atti del 1 Convegno del Gruppo di Studio sul Cinquecento francese, Fasano, Schena, 1988, p. 11-26, part. p. 19-20 (citation de lieux pertinents de Du Bellay, Le Tasse, Castiglione, Leonardo Bruni, Marin Sanudo). La balance commerciale des traductions entre les deux pays peut être calculée grâce au répertoire de J. Balsamo, G. Dotoli et V. Castiglione Minischetti (dir.), Les traductions de l'italien en français au XVI e siècle, Fasano / Paris, Schena / Hermann, 2009, et celui de V. Grohovaz, «La traduzione dal francese all'italiano nel XVI secolo. Avvio di una catalogazione delle opere a stampa (1501-1650)», dans Ugo Rozzo (dir.), La lettera e il torchio. Studi sulla produzione libraria tra XVI e XVIII secolo, Udine, Forum, 2001, p. 71-165. Ce qui frappe d'abord, c'est le nombre de traductions françaises par rapport aux italiennes. Alors que presque tous les grands écrivains italiens sont traduits en France au $\mathrm{xVI}^{\mathrm{e}}$ siècle (l'Arioste, Pétrarque, Bembo, Boccace, Dante, Sannazar, Machiavel, etc.), nous ne trouvons pas de versions italiennes des chefs-d'œuvre de Rabelais, Du Bellay, Ronsard, Maurice Scève (à l'exception d'un poème et de l'Entrée d'Henri II), Clément Marot, Marguerite de Navarre, etc.

2. Les données essentielles sur Lucantonio (ou Luca Antonio) Ridolfi, ont été réunies par : É. Picot, Les français italianisants au XVI e siècle, Paris, Champion, 1906-1907, 2 vol., vol. 2, p. 19-26; 
R. Traversa, L'attività lionese di Luc'Antonio Ridolfi, Tesi di laurea, Università di Torino, 1964-1965 [une copie à l'Archivio Storico dell'Ateneo di Torino]; E. Giudici, «Luc'Antonio Ridolfi et la Renaissance franco-italienne ", Quaderni di filologia e lingue romanze, n. s., vol. I, 1985, p. 111-150 ; M. M. Fontaine, «"Un cœur mis en gage”. Pontus de Tyard, Marguerite de Bourg et le milieu lyonnais des années 1550 », Nouvelle Revue du XVI siècle, vol. II, 1984, p. 69-89 ; R. Cooper, Le Cercle de Lucantonio Ridolfi, dans M. Clément et J. Incardona (éds), L'Émergence littéraire des femmes à Lyon à la Renaissance, Saint-Étienne, Publications de l'Université de Saint-Étienne, 2008, p. 29-50.

3. Sur ces questions, voir entre autres: J. Boucher, Présence italienne à Lyon à la Renaissance du milieu du XVe siècle à la fin du XVI e siècle, Lyon, LUGD, [1994] et Id., "Les Italiens à Lyon ", dans J. Balsamo (éd.), Passer les monts. Français en Italie - L'Italie en France (1494-1525), Paris / Fiesole, Champion / Cadmo, 1998, p. 297-312 ; A. Possenti et G. Mastrangelo (éd.), Il Rinascimento a Lione, Atti del Congresso internazionale (Macerata, 6-11 maggio 1985), Rome, Ed. dell'Ateneo, 1988 ; G. Defaux (éd.), Lyon et l'illustration de la langue française à la Renaissance, Lyon, ENS, 2003.

4. Sur cet aspect, nous nous contentons de renvoyer à l'article de I. Andreoli, «Impressions italiennes : imprimeurs, auteurs et livres italiens à Lyon au XvI ${ }^{\mathrm{e}}$ siècle ", Cahiers d'études italiennes, $n^{\circ} 27,2018$ (disponible en ligne sur <https://journals.openedition.org/cei/5167>), où on trouvera la bibliographie antérieure.

5. Voir P. Procaccioli, «Le "tre corone" a Lione. Guillaume Rouville e Lucantonio Ridolfi », dans S. D’Amico et S. Gambino Longo (éds), Le savoir italien sous les presses lyonnaises à la Renaissance, Genève, Droz, 2017, p. 223-244 ; N. Dusi, «Lucantonio Ridolfi e Francesco Petrarca. Un esegeta fiorentino a Lione ", Studi Petrarcheschi, n 20, 2007, p. 125-150; N. Bianchi, « Un altro lettore di Dante nel Cinquecento ", Studi Danteschi, n 66, 2001, p. 205-223.

6. In Lione, appresso Guillaume Rouillé, 1557 (des copies numérisées dans Gallica et dans le site de la Bayerische Staatsbibliothek). L'ouvrage a été réimprimé en 1558 ("édition rajeunie» d'après Baudrier) et plus tard dans une émission de 1560 (avec titre modifié et premier cahier réimprimé), toujours par Rouillé : voir H. Baudrier, Bibliographie lyonnaise, Genève, Slatkine Reprints, 1999 [réimp. de l'éd. de Lyon, 1895-1921], vol. IX, p. 242, 250-251, 272. Le texte est anonyme mais doit être attribué à Ridolfi (voir É. Picot, Français italianisants, ouvr. cité, vol. 2, p. 24-25 ; N. Dusi, «Lucantonio Ridolfi e Francesco Petrarca », art. cité, ainsi qu'infra note 9). Une contribution de Claire Lesage sur le Ragionamento vient de paraître : " "La forma del ben dire" : les lecteurs français du Ragionamento de Lucantonio Ridolfi (1557)», dans P. Guérin et A. Robin (éds), Boccaccio e la Francia, Florence, Franco Cesati, 2017, p. 259-270.

7. Cf. infra et n. 10.

8. In Lione, appresso Guliel. Rovillio, M.D.LX (nous avons consulté l'exemplaire Paris, Bibliothèque de la Sorbonne, RBA $8=26$, où notre texte est la troisième et dernière pièce d'un recueil factice de livres imprimés par Rouillé). L'Aretefila a été imprimée encore par Rouillé en 1562 (version numérisée sur Gallica) : voir H. Baudrier, Bibliographie lyonnaise, ouvr. cité, vol. IX, p. 265 et 286. Il s'agit d'une réémission de l'édition de 1560 avec nouvelle page de titre. Par rapport à l'édition précédente, on retrouve la même page d'Errata.

9. Saulnier se posait la question de savoir si « Lucio ne représenterait-il pas ici l'auteur lui-même, Ridolfi, qui se prénommait Luca-Antonio?» (Maurice Scève (ca.1500-1560), Genève / Paris, Slatkine, 1981 [réimpression de l'édition Paris 1948-1949], 2 vol., vol. 2, p. 164, n. 51). R. Cooper, dans « Le Cercle de Lucantonio Ridolfi », art. cité, p. 42, écrit que « Lucio est probablement Ridolfi lui-même » et M. M. Fontaine, dans « "Un cœur mis en gage" ", art. cité, p. 70 dit que « Lucio est peut-être Luc-Antonio lui-même ». L'hésitation n'a pas lieu d'être. Non seulement Ridolfi, comme Lucio, est un Toscan résident à Lyon. De plus, on peut remarquer que Lucio parle brièvement du style de Boccace parce qu'il compte en parler plus longuement dans un autre endroit (p. 111). C'est une claire référence au dialogue de Ridolfi, Ragionamento havuto in Lione..., qui paraît en 1557, peu de temps après le dialogue réel (voir aussi note suivante). Cet élément constitue une ultérieure preuve de la paternité de Ridolfi du Ragionamento (cf. note 6). 
10. Le dialogue a lieu après la mi-novembre 1556 et avant mars 1557, les terminus post quem et ante quem étant fournis par le texte, probablement vers décembre/janvier. La lettre dédicatoire de Ridolfi qui précède l'ouvrage est datée du 4 mars 1557 et parle d'un « ragionamento [...] fatto a mesi prossimamente passati» (p.3). Les devisants évoquent (p.125) la mort récente, le 14 novembre 1556, de Giovanni Della Casa. Par ailleurs, il s'agit de la période hivernale (p. 7).

11. Sur la reprise de motifs de Boccace dans les traités d'amour du $x I^{e}$ siècle, y compris dans l'Aretefila, voir M. Favaro, «Boccaccio nella trattatistica amorosa del Cinquecento e di primo Seicento ", Nuova Rivista di Letteratura Italiana, vol. XII, $n^{\circ}$ 1-2, 2009, p. 9-29, part. p. 10-12. Sur les traités qui abordent la question de l'amour de loin, dont notre dialogue, voir la thèse inédite de Brittany Kay Asaro, The Unseen Beloved. Love by Hearsay in Medieval and Early Modern Italian Literature, sous la direction de Massimo Ciavolella, University of California Los Angeles, 2013, disponible en ligne sur <https://escholarship.org/uc/item/67m5x9cj>.

12. En plus des auteurs cités ci-dessus, Lucio et Federico évoquent: Veronica Gambara, Bartolomeo Cavalcanti, Politien, Cino da Pistoia, Lodovico Martelli, Claudio Tolomei et le juriste humaniste Alessandro d'Alessandro.

13. Virgile, Plutarque, Platon, Martial, Juvénal, Ovide, Athénée, Charès de Mytilène, Cicéron, Aristote, Lucrèce, Horace, Catulle, Properce, Sénèque.

14. Ouvr. cité, p. 136-138. C'est nous qui traduisons.

15. Frédéric parle des troubadours Raimbaut d'Orange et Jaufré Rudel (ibid., p. 45-47), qu'il connaît non pas directement par leurs poèmes mais en raison de l'évocation de leurs vies dans les Trionfi de Pétrarque (Trionfo dell'Amore, IV, vv. 46-47 et v. 52). Les informations tirées de Pétrarque sont complétées par Ridolfi grâce au très répandu commentaire de Vellutello (Le volgari opere del Petrarcha con la espositione di Alessandro Vellutello da Lucca, in Vinegia, per Giovanni Antonio \& Fratelli da Sabbio, del mese d'Agosto l'anno del signore Mille cinquecento venticinque, ici f. cV ro$\left.v^{0}\right)$.

16. Ouvr. cité, p. 38 .

17. Voir entre autres: F. Montorsi, L'apport des traductions de l'italien dans la dynamique du récit de chevalerie (1490-1550), avec préface de R. Chartier, Paris, Garnier, 2015, p. 53-56 et 63-66; M. Huchon, "Amadis, "Parfaicte idée de nostre langue françoise" ", dans Les Amadis en France au XVI siècle, Paris, Presses de l'École nationale supérieure, 2000, p. 183-200; S. Cappello, «Il Discours sur les livres d'Amadis de Gaule de Michel Sevin (1548)", dans Il Romanzo nella Francia del Rinascimento, Fasano, Schena, 1996, p. 207-224 ; R. Cooper, "Notre histoire renouvelée": The Reception of the Romances of Chivalry in Renaissance France", dans S. Anglo (éd.), Chivalry in the Renaissance, Woodbridge, Boydell, 1990, p. 175-191; L. Guillerm, Sujet de l'écriture et traduction autour de 1540, Paris, Aux Amateurs de livres, 1988; M. Simonin, «La disgrâce d'Amadis ", Studi francesi, $\mathrm{n}^{\circ}$ 82, 1984, p. 1-35.

18. L. Plazenet, L'ébahissement et la délectation. Réception comparée et poétique du roman grec en France et en Angleterre aux XVI $I^{e}$ et XVII siècles, Paris, Champion, 1997, ainsi que les documents réunis dans sa propre édition d'Héliodore, L'Histoire aethiopique. Traduction de Jacques Amyot, Paris, Champion, 2008 ; M. Fumaroli, "Jacques Amyot and the Clerical Polemic against the Chivalric Novel ", Renaissance Quarterly, vol. 38, n 1, 1985, p. 22-40 [traduction remaniée de l'article dans Exercices de lecture. De Rabelais à Paul Valéry, Paris, Gallimard, 2006, p. 29-61] ; et S. Cappello, « La Prefazione di Amyot all'Histoire aethiopique di Eliodoro ", dans V. Orioles (éd.), Studi in memoria di Giorgio Valussi, Alexandrie, Edizioni dell'Orso, 1992, p. 125-146.

19. Feliciano de Silva, Amadis de Grecia [Amadis IX], Ana Carmen Bueno Serrano (éd.), Alcalá de Henares, Centro de Estudios Cervantinos, 2004.

20. Le septiesme livre d'Amadis de Gaule, histoire tres excellente d'Amadis de Grece [...], Paris, Jeanne Marnef pour Jean Longis, 1546, et Le huitiesme livre d'Amadis de Gaule, auquel sont recitées les hautes prouesses et faitz merveilleux d'Amadis de Grece, Paris, Estienne Groulleau, 1548 (les deux numérisés sur Gallica). 
21. Le neufiesme livre d'Amadis de Gaule, auquel sont contenuz les gestes de Dom Florisel de Niquee, Paris, Vincent Sertenas, 1551 (numérisé sur Gallica). C'est le premier volume de la saga qui n'est pas traduit par Herberay des Essarts.

22. Les épisodes évoqués par Ridolfi (p. 44 pour Grimarta; p. 44 pour Brimarte; p. 52 pour Darinello ; p. 61-62 et 73 pour Nichea) se lisent dans Feliciano de Silva, Amadis de Grecia, ouvr. cité, respectivement: Libro primero, chap. XIV, p. 54-55 (Grimarta), Libro primero, chap. LIX, p. 197 (Brimarte), Libro segundo, chap. cxxxI, p. 569 et suiv. (Darinello), Libro segundo, chap. XxIII, p. 296 (Nichea). Si l'on suit toujours le même ordre de citation, les passages se retrouvent dans: Le septiesme livre d'Amadis, ouvr. cité, chap. IX, f. XIX v (traduction infidèle) et chap. LIII, f. CVI v ${ }^{\circ}$; Le neufiesme livre d'Amadis, ouvr. cité, chap. II, f. III v sq. ; et Le huitiesme livre d'Amadis, ouvr. cité, chap. XVIII, f. XXXII vo-xxXIII $\mathrm{r}^{\circ}$.

23. L'historia di Amadis di Grecia, in Vinegia, per Michele Tramezzino, 1550.

24. Sur les récits chevaleresques espagnols, ou d'inspiration espagnole, imprimés en Italie, voir A. Bognolo, G. Cara et S. Neri, Repertorio delle continuazioni italiane ai romanzi cavallereschi spagnoli. Ciclo di Amadis di Gaula, Roma, Bulzoni, 2013 (en particulier S. Neri, « Il romanzo cavalleresco spagnolo in Italia », p. 85-139) ; F. Fiumara, "Tradotti pur hora»: Mambrino Roseo da Fabriano e la diffusione del romanzo cavalleresco spagnolo nell'Italia della Controriforma, Thèse de doctorat, Baltimore, John Hopkins University, 2007 et la contribution pionnière d'Henry Thomas, Spanish and Portuguese Romances of Chivalry. The Revival of the Romance of Chivalry and Its Extension and Influence Abroad, Cambridge, University Press, 1920. Sur ce corpus, voir aussi le site du Progetto Mambrino, disponible en ligne sur <www.mambrino.it>.

25. Historia di Heliodoro delle cose ethiopiche [...], tradotta nella lingua toschana da M. Leonardo Ghini, in Vinegia, appresso Gabriel Giolito de Ferrari, 1556, p. 105 (numérisé sur Google Books).

26. Héliodore, L'Histoire aethiopique. Traduction de Jacques Amyot, ouvr. cité, p. 272.

27. Ridolfi évoque l'innamoramento per fama (ouvr. cité, p. 38) de Palamède pour Yseut, épisode qui ne se trouve ni dans le roman médiéval, ni dans l'imprimé de 1489, ni dans ce remaniement qu'est le Nouveau Tristan (1554). Sauf erreur de notre part, il ne se trouve pas non plus dans les versions italiennes médiévales. Pour le $\mathrm{xvI}^{\mathrm{e}}$ siècle nous aurions voulu étendre l'enquête aux poèmes en octaves de Niccolò degli Agostini (Il primo libro dell'innamoramento di messer Tristano e di madonna Isotta, paru en 1515, et Il secondo e terzo libro di Tristano, qui date de 1520) mais, en raison de leur rareté, ceux-ci nous sont restés inaccessibles. Nous soulignons enfin que, dans la Tavola Ritonda - version italienne du Tristan (xIv ${ }^{\mathrm{e}}$ siècle, 8 manuscrits) - il y a bien un personnage qui aime de loin Yseut, c'est-à-dire Kahedin (à ce sujet voir G. Murgia, « Rappresentare il desiderio: la statua di Isotta nella Tavola Ritonda », Between, vol. 3, 2013, p. 1-23, part. p. 12-17).

\section{RÉSUMÉS}

À une époque, le $\mathrm{xVI}^{\mathrm{e}}$ siècle, où la littérature étrangère intéresse peu les esprits italiens, il existe quelques hommes de lettres de la Péninsule qui montrent une remarquable connaissance de la situation littéraire transalpine. L'un de ceux-ci est Lucantonio Ridolfi, Toscan transplanté à Lyon, qui s'illustre au milieu du siècle dans des activités éditoriales et littéraires. L'étude que nous proposons vise à reconstruire les contours de la bibliothèque française de Ridolfi, telle qu'elle peut être dessinée grâce aux multiples renvois aux textes français glissés par l'auteur dans son 
œuvre. On découvre ainsi que l'Italien Ridolfi a dressé, dans ses textes et en particulier dans l'Aretefila, l'un des panoramas les plus lucides des lettres françaises des années 1540 et 1550.

Nel Cinquecento le letterature straniere sembrano interessare poco gli Italiani. Nonostante questa tendenza, si segnalano alcune eccezioni di letterati che mostrano una notevole conoscenza della situazione transalpina. Uno di questi è Lucantonio Ridolfi, toscano trapiantato in Francia, che si illustra nel mondo dell'edizione e della cultura nella Lione della metà del 500. In questo contributo sono ricostruiti i contorni della biblioteca francese di Ridolfi, quale essa può essere disegnata a partire dai rinvii lasciati da Ridolfi nella sua stessa opera. L'analisi mostra che Ridolfi è l'autore di un panorama straordinariamente lucido della letteratura francese degli anni 40 e 50 del Cinquecento.

16th century Italy shows little interest in foreign literature. There are nevertheless exceptions to this general tendency. Some Italian men of letters display at the time a remarkable knowledge of the French literary situation. One of those men is Lucantonio Ridolfi, a Toscan who settled in Lyon, France, where he carried out editorial and literary activities in the mid of the century. The present contribution aims at sketching the French library of Ridolfi as it can be restored through the quotations Ridolfi inserted in his own works. The analysis show that the Italian conceived an extraordinarily lucid panoramas of the French literature of the 1540s and 1550s.

\section{INDEX}

Parole chiave : Lucantonio Ridolfi, letture francesi, studi di ricezione, Aretefila, Ragionamento, Lione, italianismo, Marguerite de Bourg, romanzi di cavalleria, sonetto in Francia

Mots-clés : Lucantonio Ridolfi, lectures françaises, études de réception, Aretefila, Ragionamento, Lyon, italianisme, Marguerite de Bourg, romans de chevalerie, sonnet en France

Keywords : Lucantonio Ridolfi, French readings, reception studies, Aretefila, Ragionamento, Lyon, French Italianism, Marguerite de Bourg, chivalry romances, sonnet in France

\section{AUTEUR \\ FRANCESCO MONTORSI}

Université Lyon 2

francesco.montorsi@univ-lyon2.fr 